\title{
Synthetic Vision Displays for Planetary and Lunar Lander Vehicles
}

\author{
Jarvis (Trey) J. Arthur, III, Lawrence J. Prinzel, III, Steven P. Williams, Kevin J. Shelton, Lynda J. Kramer, Randall E. Bailey \\ NASA Langley Research Center \\ Hampton, VA
}

Robert M. Norman

Boeing Phantom Works

Hampton, VA

Aviation research has demonstrated that Synthetic Vision (SV) technology can substantially enhance situation awareness, reduce pilot workload, improve aviation safety, and promote flight path control precision. SV, and related flight deck technologies are currently being extended for application in planetary exploration vehicles. SV, in particular, holds significant potential for many planetary missions since the SV presentation provides a computer-generated view for the flight crew of the terrain and other significant environmental characteristics independent of the outside visibility conditions, window locations, or vehicle attributes. SV allows unconstrained control of the computer-generated scene lighting, terrain coloring, and virtual camera angles which may provide invaluable visual cues to pilots/astronauts, not available from other vision technologies. In addition, important vehicle state information may be conformally displayed on the view such as forward and down velocities, altitude, and fuel remaining to enhance trajectory control and vehicle system status. The paper accompanies a conference demonstration that introduced a prototype NASA Synthetic Vision system for lunar lander spacecraft. The paper will describe technical challenges and potential solutions to SV applications for the lunar landing mission, including the requirements for high-resolution lunar terrain maps, accurate positioning and orientation, and lunar cockpit display concepts to support projected mission challenges.

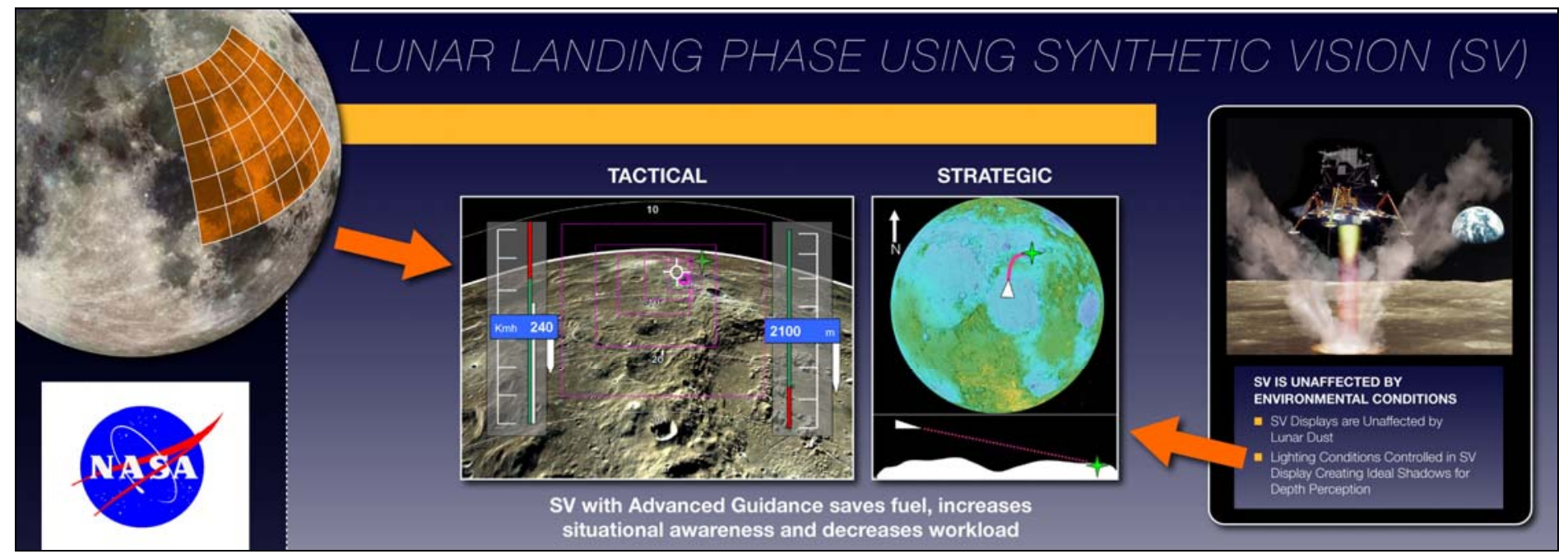

Figure 1. NASA Lunar Lander Synthetic Vision Display Concept

\section{Introduction}

$\mathrm{SV}$ is a computer-generated image of the external scene topography from the perspective of the flight deck that is derived from vehicle attitude, high-precision navigation solutions and a database that includes terrain and may include obstacles, trajectory information, relevant cultural features, and other data (Figure 1). The SV display is unaffected by outside weather and environmental conditions (e.g., fog, clouds, dust storms) and thus, provides a clear day view regardless of the available outside visibility. Though there is no weather on the moon, thrusters can create a dust cloud that can significantly reduce visibility during a critical phase of the flight. Further, sun angles can create visually powerful shadow effects which may cause the loss of depth cues, translational velocities, and landing zone awareness for the flight crew.

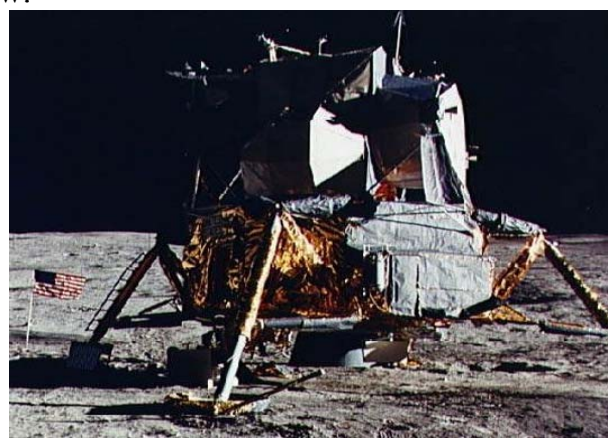

Figure 2. Apollo Lunar Module 


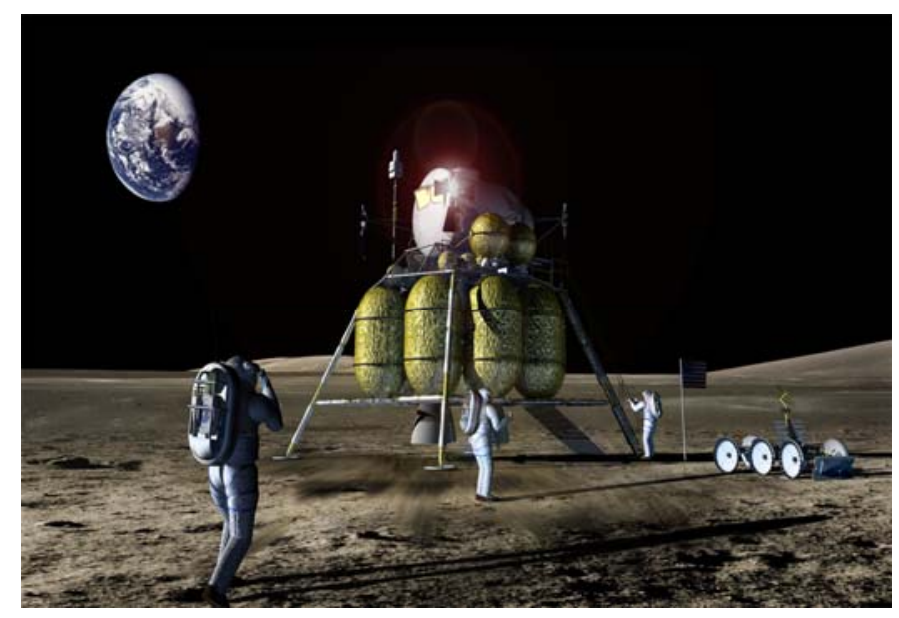

Figure 3. NASA Concept Lunar Lander

The initial design philosophy for a lunar lander flight deck should enhance the human's awareness of the vehicle state and reduce the workload of the task. It is possible to completely automate the entire landing process as evinced by Mars rovers. However the human is best at assessing and adapting to a given situation, particularly in reaction to unplanned events or failure conditions. Nonetheless, in order to make sound decisions, the human operator must understand the current state of the vehicle and not be task-saturated. In aircraft, situation awareness is achieved through cues out-thewindow as well as forming a mental model based on the aircraft instruments. SV research in aviation has showed an increase in situation awareness with reduced workload by enhancing the cockpit displays to be more intuitive to interpret. The goal is to provide enough information to keep the astronaut engaged without being task overloaded so that the current situation and future states are more quickly and accurately understood. This allows for sound decision making during critical moments such as the landing phase.

To investigate the potential benefits of a SV-enhanced flight deck for spacecraft, the lunar landing mission was explored for application (Figures 2-3). The Apollo lunar landings were an extraordinary achievement and required highly skilled and trained pilots. These astronauts were selected and trained to navigate a new vehicle in an unknown environment and adapt in the face of numerous potential failure modes and uncertainty conditions with only rudimentary, basic flight instrumentation. The flight instrumentation of the Apollo Lunar Excursion Module (LEM) consisted mainly of "electromechanical" gauges (Figure 4) which was the typical technology for aircraft in the early days of NASA. The basic gauges of the Apollo LEM flight deck required pilots (astronauts) to extract and integrate information from several instruments to form a mental model of the situation. This task required highly skilled pilots with extensive training, coupled with operational profiles which required that the pilot visually see the landing zone for extended periods of time.

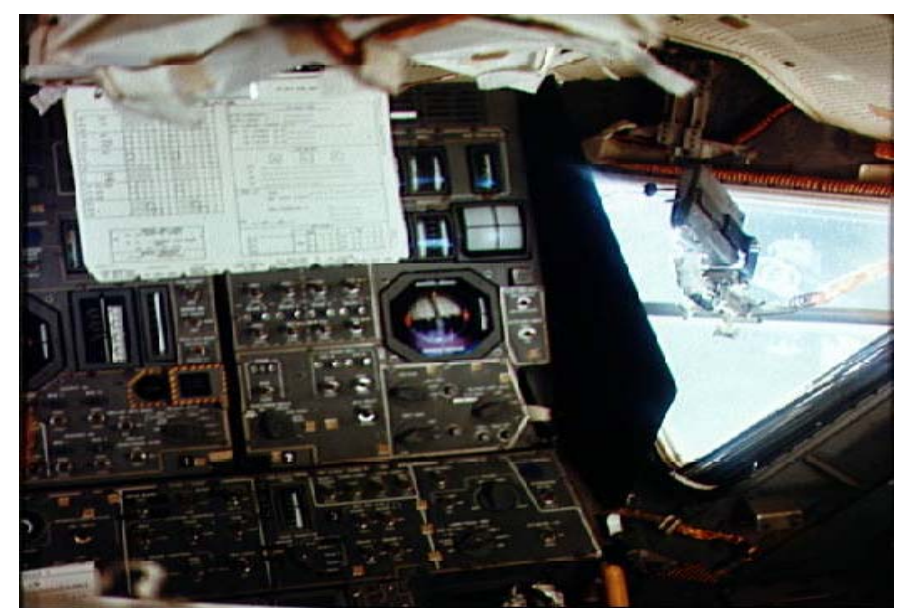

Figure 4. Apollo Lunar Module Cockpit Displays

As technology has advanced, the electro-mechanical gauges, typical for a 1970s aircraft cockpit, are being replaced with glass cockpits. (Even the Space Shuttle has been upgraded to a glass cockpit; in this case, however, the electro-mechanical gauge information is simply drawn on the new displays, not taking full advantage of current graphical capabilities.) As computation and graphics display power has increased, the ability to render complex scenes in real-time offered the opportunity to redesign cockpit displays from an optimized human factors standpoint. The ability to create intuitive displays of the vehicle state in a flight deck display has become a reality; that same realization for spacecraft is the focus of this research.

Aviation research associated with SV has demonstrated that these intuitive displays are superior to traditional displays especially in high workload situations. The landing phase was identified by Neil Armstrong as being the most difficult part of Apollo 11, therefore, this project focused on the descent stage to landing. The Apollo 15 landing site was chosen for study because of the interesting terrain features and the availability of higher resolution lunar terrain data, for this landing site.

The importance of pilot visibility was not only emphasized by trajectory design and window definition, but also, by conducting the Apollo landing task only at specific times and locations to provide optimal sun light on the landing site. The mission was designed around lighting conditions that would create shadows that would provide optimal depth perception. These optimal lighting opportunities typically lasted about a week. If the opportunity was missed, the next opportunity to land would not be for another month. As envisioned, future space operations are likely to require frequent trips to the lunar surface emphasizing the importance of providing new technological capabilities for unfettered moon surface landings. 


\section{SV Enhanced Lunar Lander Displays}

With SV, the designer controls the computer-generated scene lighting, terrain coloring, and virtual camera angles. The visual cues for the landing site in the SV are independent of the sun-angle. In addition, important vehicle state information such as forward and down velocities, altitude, and fuel remaining can be overlaid directly onto the terrain display to significantly ease pilot interpretation of the data and enhance situation awareness.

Another advantage for using SV enhanced displays is that advanced precision guidance can be intuitively integrated into SV displays. With one of the US Space Exploration Policy goals to return to the moon, frequent missions to the moon will require precise landing of vehicles (possibly within 10 meters accuracy). It is currently planned to have several habitat modules, power generators, storage and surface mobility units. Therefore, the landing task will involve not only landing on suitable terrain but avoidance of man-made obstacles and approach procedures to avoid over-flight and potential contamination.

Moreover, while SV displays provide for intuitive, easily interpretable real-time cockpit displays, they have the potential to be used on-board in mission-rehearsal or during near real-time landing site re-planning tasks. The displays would enable the ability to preview the planned trajectory and directly visualize its relationship to the terrain, the landing site, and fuel status prior to flying the mission. Contingency planning and scenarios can be rehearsed and then executed directly on the same displays; thus, the transfer of training is direct. The ability of the crew to handle emergency or failure conditions, particularly the aspect of fuel-/energymanagement, will be critical. While this premise has not yet been tested, aviation-domain applications of this technology have been shown to be beneficial.

\section{Flight Deck Design Development}

Concepts for lunar lander flight deck display designs are being investigated by the Crew-Vehicle Interface team at the Langley Research Center. This multi-year effort involves the application of "equivalent vision" technologies to assist in the planetary exploration application domain. These state-of-theart "Aeronautics" technologies should play a crucial role in future manned spacecraft flight deck design, just as they are emerging as key technologies in commercial, business, and general aviation. The following concepts showcase some initial concepts applying SV technology which are likely not representative of the final concepts, but highlight the significant potential for "equivalent vision" technologies in the lunar landing mission.

Tactical Display. Tactical displays contain guidance and vehicle state information to aid the pilot in immediate navigation. The prototype lunar display examples shown here are modifications to aviation-domain concepts which may not necessarily be appropriate to the lunar landing task. Research is on-going to develop appropriate display concepts to support this new mission. The tactical display development was by no means "optimized" but represented a basis from which to quickly evaluate the feasibility of the lunar SV concept. For instance, the first challenge for the lunar lander application is the extreme attitude variations during the approach and landing and its critical role on deceleration and precision landing. The display development began initially with a velocity-vector centered display concept but was eventually replaced with an attitude-centered display. The velocity vector display was difficult to interpret in hover mode as the velocity vector can have a large drift and may induce spatial disorientation. Therefore, an attitude centered display, albeit with the ability to move the SV camera position, was investigated. This feature proved especially useful during the hover maneuver which allowed the landing spot to be viewed in the SV display. The tactical display was further enhanced by adding symbology. The prototype displays in Figures 5 and 6 show the conceptual primary flight display and navigation display during a lunar landing.

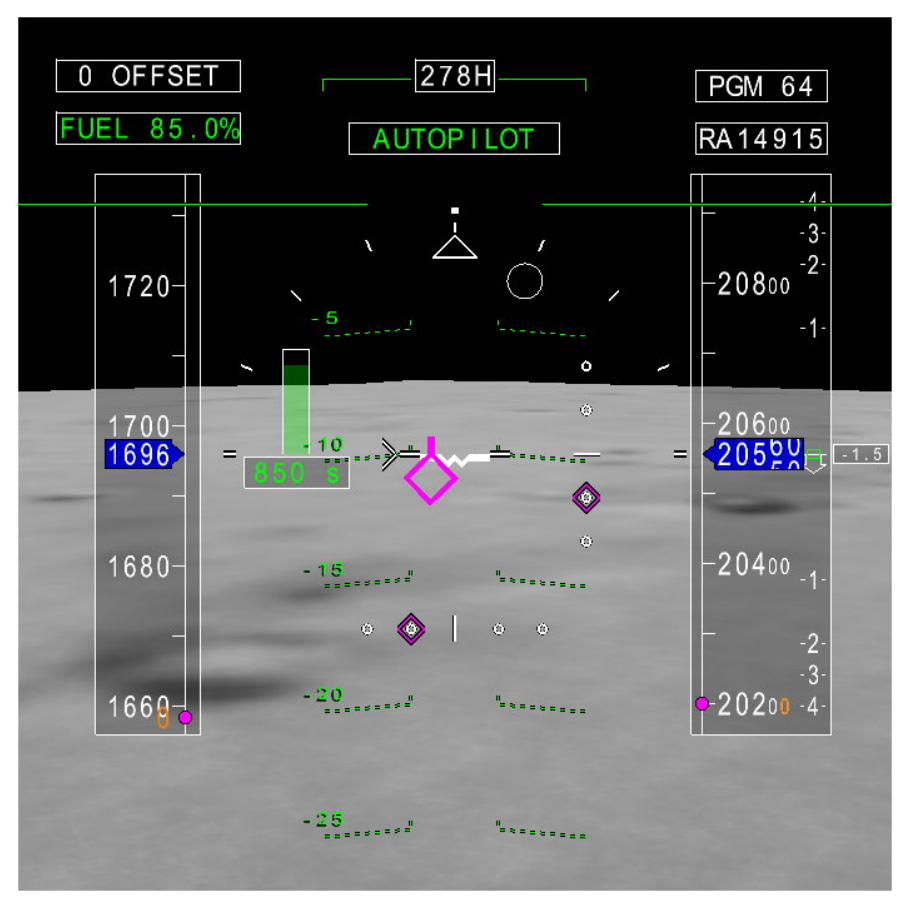

Figure. 5. SV Lunar Lander Primary Flight Display (PFD) 


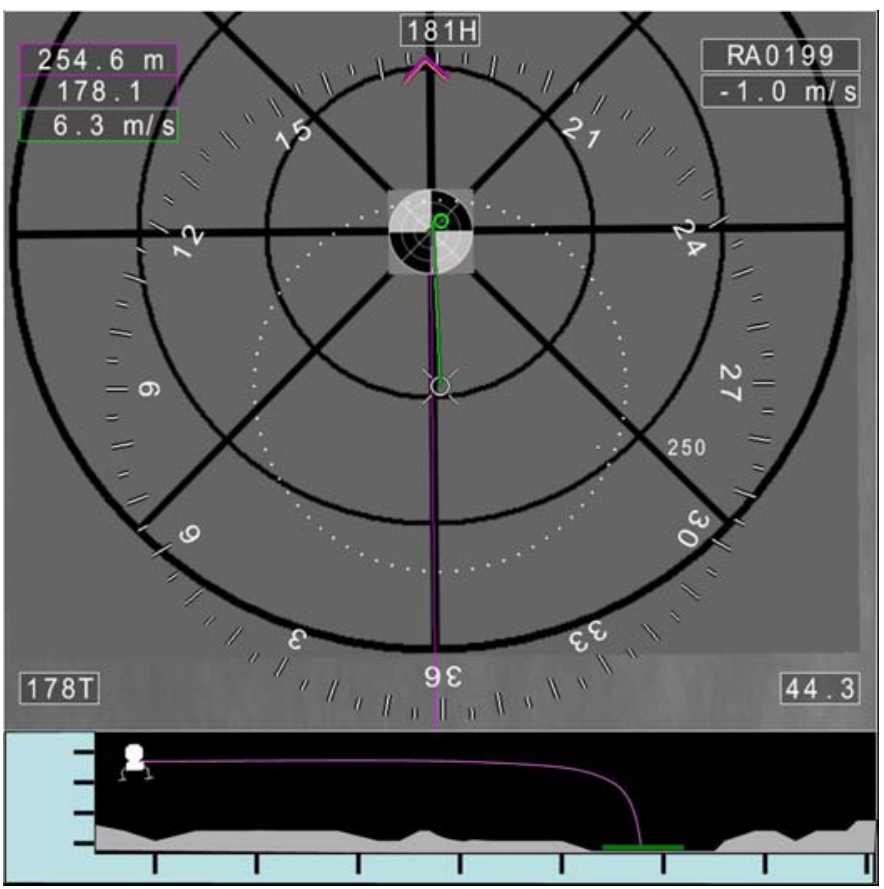

Figure 6. SV Lunar Lander Navigation Display (ND) During Touchdown

Navigation Display. Initial navigation display concepts are also being developed using the "aviation-domain" as a point of departure in our investigations. In Figure 7, a simple two dimensional, top down view with own-ship position located at the center of the display is shown, which might be typical of a Synthetic Vision Navigation Display for aircraft. The synthetic lunar terrain is layered under the symbology. A touchdown zone is drawn centered on the designated touchdown site and has a radius of 500 meters. Similarly, a touchdown pad is also drawn centered at the designated touchdown site and has a radius of 50 meters. The navigational display can be oriented track-up or heading-up as required by the pilot.

The lunar navigation display symbology is modeled after modern aircraft displays with the exception of the zero velocity symbol. The zero horizontal velocity symbol (green circle) is the point where the vehicle's horizontal velocity will reach zero, based on its current lateral and longitudinal accelerations. This provides guidance to set pitch angle for braking after the vehicle has rolled to the upright position in the final minutes of the approach before landing. During this approach phase, the vehicle is pitched back (nose up) and is slowing the approach speed. The pilot pitches more forward to reduce braking and the circle moves further down range. Likewise, when the pilot pitches further aft (increasing braking), the circle comes closer to the own ship location. To date, this raw data information provides a seemingly simple task to center the circle on the desired touchdown spot during the approach.

There are several text boxes surrounding the compass ring. The color of the box edge correlates to the symbol on the display. The magenta boxes show the range and bearing angle to the designated touchdown site, and the magenta carat on the compass ring indicates the same angle. The green box shows the current speed and is the same color as the trend vector and zero velocity circle. Radar altitude is the height above the lunar surface and is shown in the upper right text box. Just below the radar altitude box is the vertical velocity text box. In the lower left of the display an orange box gives the selected orientation angle (Track angle if heading-up mode is selected, heading angle if track-up mode is selected). The orange carat indicates this angle.

Exocentric Display. The exocentric view is a 3D representation of the lunar lander, its current velocity vector, and the surrounding synthetic terrain (Fig. 8). The exocentric view display was modeled from aircraft displays developed in previous research. The view is from a wingman perspective (chase plane) and is positioned to the rear quarter of own ship position. The view angle is manipulated such that all points of interest are within the view space; e.g., the lander, and the projected touchdown location. The attitude of the lander is correctly depicted based on real-time vehicle state data. Much of the traditional 2D navigation symbology is retained on this display, such as the compass ring with bearing and track angles, as well as the altitude, target data, and velocity text boxes.

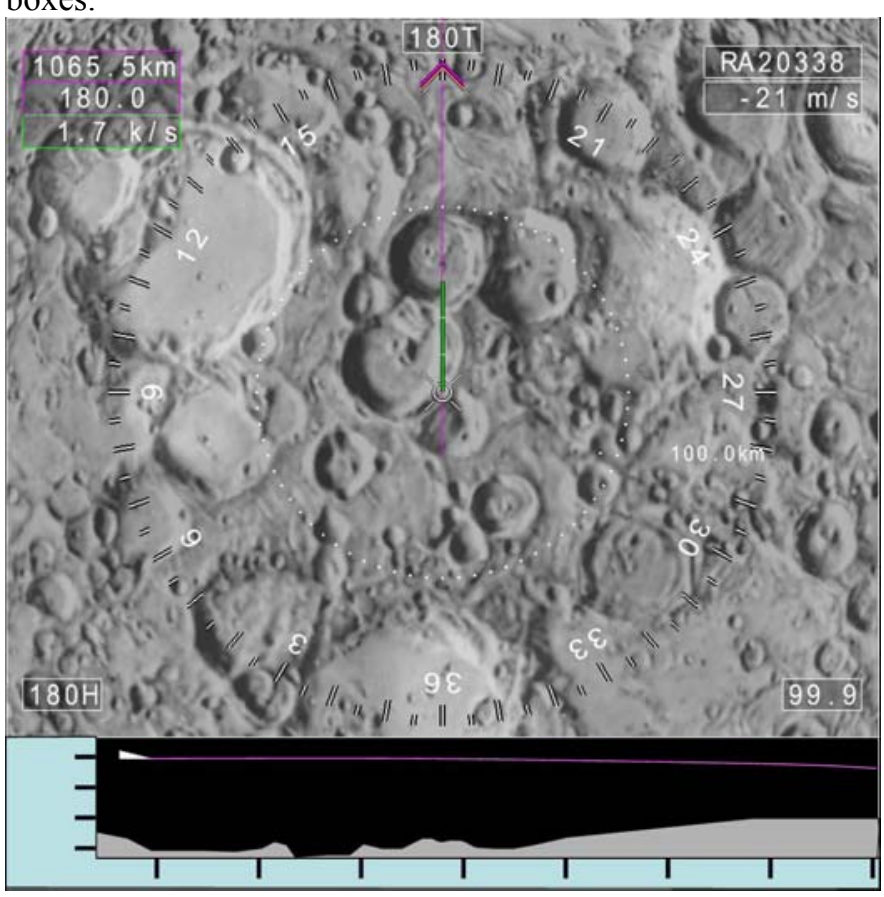

Figure 7. SV Lunar Lander ND with Symbology 


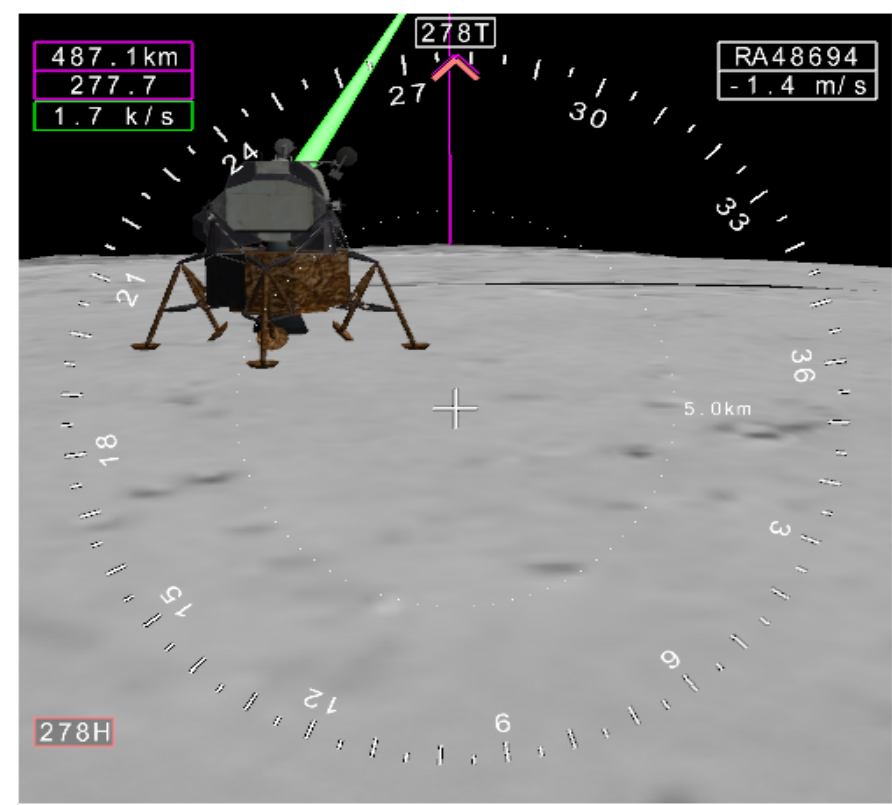

Figure 8. SV ND Exocentric View Approaching Landing Target Zone

\section{Lunar Model Development}

One essential component to SV cockpit displays is an onboard terrain database. Currently, the available lunar digital elevation data are not of sufficient resolution if we assume that aviation standards (i.e., RTCA DO-276 requirements) apply to lunar landing applications. Clementine Project (1994) data is available for the lunar terrain but it is on the order of 4 terrain posts (i.e., data points of latitude, longitude, and elevation) per degree. For our demonstration, higher resolution for global coverage terrain data were obtained for the Apollo 15 landing site, are being used. This higher resolution data is 64 terrain posts per degree which, at the lunar equator, is approximately one data point every 500 meters. RTCA DO-276 requirements would suggest terrain resolution of approximately one data point every 10 meters, with vertical accuracies less than 1 meter to support Category II/III landing operations. In the polar regions of the moon (the area where new missions are being planned), the Clementine mission did not use laser altimeter data (above $\mathrm{N} 60^{\circ}$ and below $\mathrm{S} 60^{\circ}$ ). Stereo-pair imagery was used for generating elevation data in the Apollo Lunar Module Display polar regions and this data suffers with an estimated vertical accuracy of approximately 130 meters. Planned lunar missions, such as the Lunar Reconnaissance Orbitor (LRO) set to launch in Fall 2008, will provide 5-meter data for terrain and 1-meter data for imagery. This data resolution would be sufficient, based on RTCA DO-276, to support Category III aviation operations.

One of the problems the Apollo crews faced was the determination of altitude as a sole-source or back-up, redundant source for comparison against other instrumentation sources. Given good terrain elevation models, the lunar model can be used in deriving height above the terrain if the three dimensional position of the spacecraft is accurately known.
During the initial lunar orbits, a sextant was used to verify altitude. This was because the onboard radar system had a limited range and a fixed antenna which only worked when pointed towards the moon. Therefore, under certain altitudes and attitudes, the radar altimeter could not be used. However, with SV, the lunar model can be used in deriving height above the terrain if the three dimensional position of the spacecraft is accurately known. SV is not limited by the distance or antenna placements constraints of the radar altimeter. The accuracy of the $\mathrm{SV}$ derived altitude is dependent on an accurate navigation solution and the resolution of the terrain database.

\section{Initial Evaluations}

Test pilots and astronauts did preliminary evaluations of the SV display concepts. The evaluations consisted of a short training period followed by simulated flights of the lunar lander. No questionnaires or data were collected, just anecdotal comments. Test pilots with at least 2 days of training were able to routinely land within 1 meter of the intended landing point. Two shuttle astronauts did preliminary evaluations of the displays and were also able to land with good accuracy to the intending landing point with just a few minutes of training. The developed displays, therefore, appear to enable pilots to make highly accurate, safe landings, even in their "un-optimized" state.

Future areas of research include improved symbologies, the required accuracy of the terrain database, and the need for external sensors.

\section{References}

Baxes, G. A. \& Linger, T. (2006). On-the-sphere block-based 3D terrain rendering using a wavelet-encoded terrain database for SVS. Enhanced and Synthetic Vision 2006 6226(1). 622605. SPIE.

Chin, G., Brylow, S., Foote, M., Garvin, J., Kasper, J,. Keller, J., Litvak, M., Mitrofanov, I., Paige, D., Raney, K., Robinson, M., Sanin, A., Smith, D., Spence, H., Spudis, P., Stern, S. \& Zuber, M. (2007). Lunar reconnaissance orbiter overview: The instrument suite and mission. Space Science Paper 129, 391-419.

Cummings, M., Wang, E., Smith, C., Marquez, J., Duppen, M. \&. Essama, S., (2005). Conceptual human-system interface design for a lunar access vehicle. Tech. Rep. HAL2005-04, MIT for Draper Labs, Cambridge, MA

NASA (2007). Draft constellation programmatic environmental impact statement. Washington, DC.

NASA (2004). The vision for space exploration.

Slayton, D. (1969). Apollo: Past, present and future. 13th Symposium Proceedings. The Society of Experimental Test Pilots. Beverly Hills, CA.

Williams, S. P., Arthur III, J.J., Shelton, K. J., Norman, R. M. \& Prinzel III, L. J. (2008). Synthetic vision for lunar and planetary landing vehicles. SPIE. Synthetic and Enhanced Vision 2008. 